\title{
TEM Study of Multi-Layer GaSb/GaAs QD Structures for Intermediate Band Solar Cells
}

Andrew J. Martin*, Guang Ran**, Kai Sun*, Lumin Wang****, Joanna Millunchick*

* Department of Materials Science and Engineering, University of Michigan, Ann Arbor, MI 48109

** Department of Nuclear Engineering and Radiological Sciences, University of Michigan, Ann Arbor, MI 48109

Interest in solar energy has continued to grow as more efficient sources of renewable energy are continually developed. Intermediate band solar cells (IBSCs) are of particular interest due to their high theoretical efficiency limit, which at $>63 \%$ is more than double that of a conventional homojunction cell [1]. The type-II band offset of the $\mathrm{GaSb} / \mathrm{GaAs}$ quantum dot (QD) system is believed to be advantageous for IBSCs because of its ability to more efficiently separate charge carriers due to its band shape [2]. Furthermore, Huffaker et al. have demonstrated improved infrared spectral response from $\mathrm{GaSb} / \mathrm{GaAs} \mathrm{QD}$ solar cells [3]. Cross-sectional transmission electron microscopy (TEM) of multi-layer $\mathrm{GaSb} / \mathrm{GaAs}$ QD structures with more than two layers has not been reported in literature [4]. The purpose of this paper is to compare the formation of GaSb QDs on $t$ ifferent startinG sbrfases to eoamine hoe $t$ ot shat eesireeant $t$ efest strbstbre are affestet $t$

Two multi-layer $\mathrm{GaSb} / \mathrm{GaAs}$ QD structures with eleven layers were grown by molecular beam epitaxy (MBE) on $\mathrm{N}^{+} \mathrm{GaAs}(001)$ substrates. Following oxide desorption, a $500 \mathrm{~nm}$ GaAs buffer layer was grown on both samples at $\mathrm{T}=590^{\circ} \mathrm{C}$. The GaAs spacer layer between $\mathrm{GaSb}$ QD layers was $20 \mathrm{~nm}$ thick. The top layer of QDs for both samples remained uncapped for analysis by atomic force microscopy (AFM). The Ga, Sb, and As fluxes were $0.3,0.6$, and 2.7 monolayers per second $\left(\mathrm{ML} \mathrm{s}^{-1}\right)$ as measured by reflective high-energy electron diffraction oscillations. For sample A, QDs were grown on a GaAs $2 \times 4$ surface reconstruction at $\mathrm{T}=460^{\circ} \mathrm{C}$ by deposition of $2.3 \mathrm{MLs}$ of $\mathrm{GaSb}$. Sample A was quenched under 0.3 ML s ${ }^{-1} \mathrm{Sb}$ flux. Sample B was grown on an Sb-rich GaSb 2x8 surface reconstruction, which was achieved in a similar manner as described by Huang et al. [5]. After achieving the $2 \times 8$ at $\mathrm{T}=580^{\circ} \mathrm{C}$, the temperature was decreased to $\mathrm{T}=460^{\circ} \mathrm{C}$ under $0.6 \mathrm{ML} \mathrm{s}^{-1}$ $\mathrm{Sb}$ flux for deposition of $1 \mathrm{ML}$ of GaSb for QD formation, followed immediately by a $20 \mathrm{~nm}$ GaAs cap at the same temperature. Due to the Sb-rich GaSb 2x8 starting surface, the total amount of GaSb was nominally the same in the two samples. This process was repeated for each layer of sample B. Sample B was quenched under no overpressure because surface dots formed on the GaSb $2 \times 8$ reconstruction did not tend to dissociate in the absence of $\mathrm{Sb}$ the way the dots on the GaAs $2 \times 4$ did.

Fig 1a and $1 \mathrm{~b}$ are cross-sectional BF-TEM images of samples A showing the multi-layer QD structure. Fig 1c and 1d are cross-sectional high angle annular dark field (HAADF) images of sample B. Neither sample demonstrated the vertical QD alignment often seen in stacked QD structures $[6,7]$ due to the large interlayer thickness, which was beyond the correlated region for QD alignment [8]. Inverse fast Fourier transform (IFFT) analysis of the HR images of both samples (Fig $1 \mathrm{~b}$ and $1 \mathrm{~d}$ ) revealed a few dislocations in the vicinity of the QD interface in sample A, but none in sample B. AFM of the uncapped surface dots shown in Fig 2a and 2b confirmed that QDs for sample B were shorter and larger in diameter. Sample A QDs had an aspect ratio of $12.4 \pm 3.1$ as compared to $15.6 \pm 3.4$ for sample B. Photoluminescence was also measured and will be reported on elsewhere. 
References:

[1] G. Wei, et al., Appl. Phys. Let. 91, 223507 (2007).

[2] L. Cuadra, A. Marti, A. Luque, Physica E 14 (2002) 162-165.

[3] R.B. Laghumavarapu, et al., Appl. Phys. Let. 90, 173125 (2007).

[4] C. Jiang, et al., J. of Cryst. Grth. 301/302, 828-832 (2007).

[5] S. Huang, G. Balakrishnan, D. Huffaker, J. of Appl. Phys. 105, 103104 (2009).

[6] L. Bouzaiene, et al., Appl. Phys. A 79 587-591 (2004).

[7] Z.R. Wasilewski, S. Fafard, J.P. McCaffrey, J. of Cryst. Grth. 201/202 1131-1135 (1999).

[8] S. Kiravittaya, A. Rastelli, O. Schmidt, Rep. Prog. Phys. 72046502 (2009).

[9] A.J.M and J.M. acknowledge the support of EFRC Grant Number DE-SC000957.


FIG. 1. (a) BF image of sample A showing the multi-layer GaSb/GaAs QD structure and (b) HR BF image of the QD shown in the small box in (a). (c) and (d) HAADF images of sample B including a HR image of the QD shown in the small box in (c).
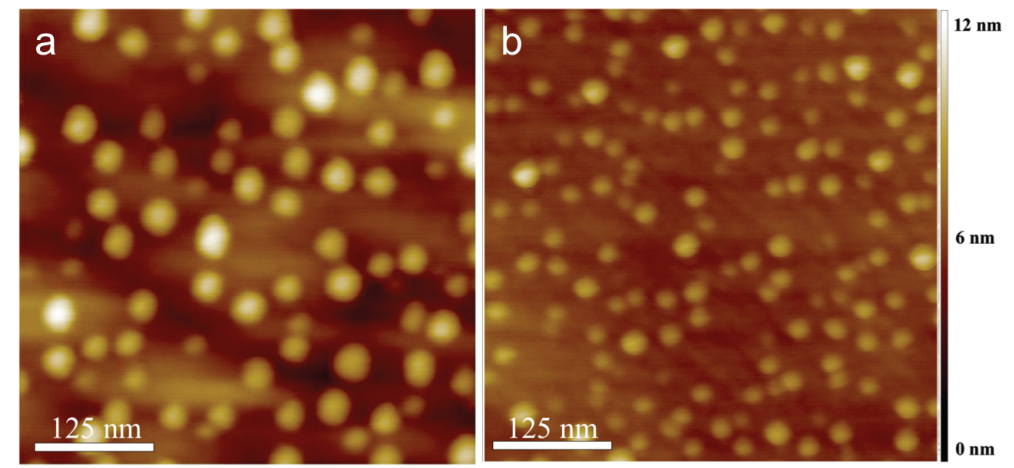

FIG. 2. (a) and (b) AFM images of the uncapped surface of samples A and B, respectively. 